\title{
Electromagnetic resonances in individual and coupled split-ring resonators
}

\author{
Philippe Gay-Balmaz and Olivier J. F. Martin ${ }^{\text {a) }}$ \\ Electromagnetic Fields and Microwave Electronics Laboratory, Swiss Federal Institute of Technology, \\ ETH-Zentrum, ETZ, CH-8092 Zurich, Switzerland
}

(Received 27 December 2001; accepted for publication 10 June 2002)

\begin{abstract}
We study experimentally and numerically the electromagnetic resonances in split ring resonators (SRRs), around $1 \mathrm{GHz}$. For an individual SRR, we show that both electric and magnetic fields can induce resonances, the magnetic one being the strongest. The utilization of such resonant structures as efficient microwave filter is also demonstrated. The coupling between two or more SRRs can be quite complex and strongly depends on their geometrical arrangement. For small separation distances, very strong coupling, leading to sharp resonances with high quality factors are observed. In that case a magnetic field circulation which connects neighboring elements is established. The practical implications of these results for the fabrication of a left-handed metamaterial are discussed. (C) 2002 American Institute of Physics. [DOI: 10.1063/1.1497452]
\end{abstract}

\section{INTRODUCTION}

Recently, split ring resonators (SRRs) have been successfully used to build left-handed metamaterials. ${ }^{1-3}$ In such a composite material, the electromagnetic waves behave in a reversed manner and many peculiar effects arise: a convergent lens becomes divergent (and vice versa), the Doppler shift, and the Cherenkov radiation wavelength are reversed. These surprising properties were already postulated more than three decades ago by Veselago, ${ }^{4}$ but they have only been demonstrated experimentally very recently, ${ }^{3}$ and new effects based on left-handed materials are just being anticipated. ${ }^{5-8}$

A left-handed material requires simultaneous negative permittivity $\varepsilon$ and negative permeability $\mu$. At microwave frequencies, the former can be obtained with very thin nanowires that behave like a low frequency plasmonic medium with plasma frequency $\omega_{\mathrm{p}} \cdot{ }^{9,10}$ On the other hand, a negative permeability over a given frequency range can be realized in a periodic array of SRRs. ${ }^{11}$ While negative permittivity can be obtained in the nanowires for all frequencies smaller than $\omega_{\mathrm{p}}$, the negative permeability only occurs in a narrow frequency band, on the high-frequency side of the SRRs resonance. This resonance therefore determines the range where the material will simultaneously have negative $\varepsilon$ and $\mu$, and exhibit a left-handed behavior. The interaction between SRRs and its influence on the band where $\mu<0$ is therefore essential for the practical realization of a lefthanded material.

A typical SRR is shown in Fig. 1; it is made of two concentric rings, each interrupted by a small gap. This gap strongly decreases the resonance frequency of the system. Like this, it is possible to obtain a resonant structure with dimensions only a tenth of the corresponding wavelength, compared to a quarter of a wavelength for a closed, uninterrupted ring.

\footnotetext{
a) Author to whom correspondence should be addressed; electronic mail: martin@ifh.ee.ethz.ch
}

The response of an infinite periodic arrangement of SRRs has been investigated in detail by Pendry et al. ${ }^{11}$ In this article we investigate experimentally and numerically the behavior of an individual SRR, as well as the coupling between several SRRs. Our purpose is to study the character of this coupling and determine the optimal configuration to enhance it.

The article is organized as follows: The experimental setup is described in Sec. II, the computational model outlined in Sec. III, individual and interacting SRRs are studied in Sec. IV, and the results are summarized in Sec. V.

\section{EXPERIMENTAL SETUP}

The resonance frequency of a SRR scales directly with its dimensions. The measurements and simulations in the present work are performed around $1 \mathrm{GHz}$ (wavelength $\lambda$ $\approx 30 \mathrm{~cm}$ ), which corresponds to large structures that are easily fabricated. The investigated SRR and its dimensions are described in Fig. 1. We consider a square SRR geometry as it is particularly simple to make and has been extensively used in left-handed metamaterials. ${ }^{1-3}$ For the experiment, individual SRRs were cut in $50 \mu \mathrm{m}$ thick self-adhesive aluminum foil and stuck onto a foam which permittivity $\varepsilon$ at microwave frequencies is close to that of air (Rohacell HF 51, $\varepsilon=1.07)$.

One or several SRRs were then placed in the middle of an R9 rectangular waveguide (section 247.65 $\times 123.82 \mathrm{~mm}^{2}$, operation range $\left.0.76-1.15 \mathrm{GHz}\right)^{12}$ and the scattering parameters measured using a network analyzer (HP 8753B). The waveguide was excited with a $\mathrm{TE}_{10}$ mode. For the comparison with theoretical results, it is important to keep in mind that the waveguide implicitly imposes periodic boundary conditions on the system. The structure under study is therefore effectively repeated periodically.

\section{MODEL}

For the analysis of the SRR depicted in Fig. 1, we adopt the Green's tensor technique. The main advantage of this 
(a)

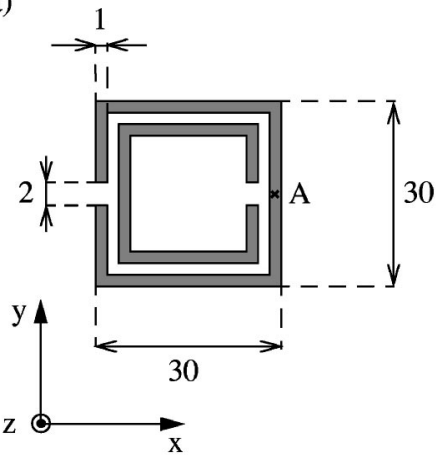

(b)

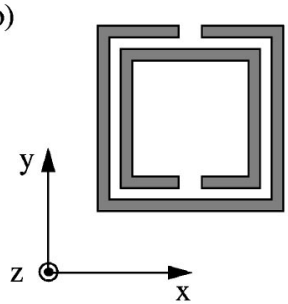

Parallel polarization

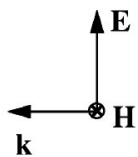

Perpendicular polarization

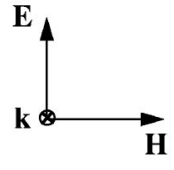

Parallel polarization

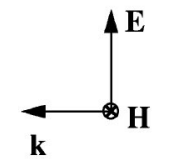

Perpendicular polarization

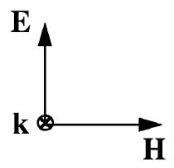

FIG. 1. Split ring resonator (SRR) used in this study, all dimensions in mm. The gap between inner and outer rings is $1 \mathrm{~mm}$. The SRR is illuminated with a plane wave propagating in the $\mathbf{k}$ direction and two different illumination polarizations are considered: parallel polarization, where the magnetic field $\mathbf{H}$ is parallel to the SRR $z$ axis and perpendicular polarization, where it is normal to that axis. (a) and (b) Two different orientations of the SRR arms with respect to the electric field $\mathbf{E}$ are investigated.

approach lies in the fact that only the conductors must be discretized, a more complex background like a surface or a layered substrate being taken into account in the Green's tensor. $^{13,14}$

The objective of the simulations is to determine the surface currents $\mathbf{J}$ induced in the conductors forming the SRRs, when the structure is illuminated with an incident field $\mathbf{E}^{\mathrm{i}}$. These currents must satisfy the following boundary conditions on the surfaces $S$ of the conductors: ${ }^{15}$

$$
\mathbf{n}(\mathbf{r}) \times\left[\mathbf{E}^{\mathrm{s}}(\mathbf{r})+\mathbf{E}^{\mathrm{i}}(\mathbf{r})\right]=Z(\mathbf{r})[\mathbf{n}(\mathbf{r}) \times \mathbf{J}(\mathbf{r})],
$$

where $\mathbf{E}^{\mathrm{s}}$ is the scattered field produced by the current, $Z$ a surface impedance which allows to take into account the finite conductivity of the conductor, and $\mathbf{n}$ the outward unit normal to the conductor surface.

Using a mixed potential approach, ${ }^{15}$ the scattered field can be written in terms of potentials via the Green's tensor. In this way Eq. (1) can be written as

$$
\begin{aligned}
\mathbf{n}(\mathbf{r}) \times \mathbf{E}^{\mathrm{i}}(\mathbf{r})= & Z(\mathbf{r}) \mathbf{n}(\mathbf{r}) \times \mathbf{J}(\mathbf{r})+\mathbf{n}(\mathbf{r}) \\
& \times\left[j \omega \iint_{S} \overline{\overline{\mathbf{G}}}_{\mathrm{AJ}}\left(\mathbf{r}, \mathbf{r}^{\prime}\right) \cdot \mathbf{J}\left(\mathbf{r}^{\prime}\right) d s^{\prime}\right. \\
& \left.-\frac{1}{j \omega} \nabla \iint_{S} G_{V}\left(\mathbf{r}, \mathbf{r}^{\prime}\right) \nabla \cdot \mathbf{J}\left(\mathbf{r}^{\prime}\right) d s^{\prime}\right],
\end{aligned}
$$

where $\overline{\mathbf{G}}_{\mathrm{AJ}}$ is the vector potential Green's tensor and $G_{V}$ the scalar potential Green's function. Equation (2) represents the mixed-potential integral equation for the conductor bodies with surface $S$. To solve this equation numerically, the method of moment is applied and the unknown currents $\mathbf{J}(\mathbf{r})$ expanded in a series of $N$ basis functions $\mathbf{f}_{j}(\mathbf{r})$ defined on rectangular elements:

$$
\mathbf{J}(\mathbf{r})=\sum_{j=1}^{N} \alpha_{j} \mathbf{f}_{j}(\mathbf{r}) .
$$

First order Lagrange polynomial, defined on two adjacent rectangular elements, are used as basis functions. For a typical SRR about 70 discretization elements are required to obtain an accurate representation of the current.

To compute the unknown coefficients $\alpha_{j}$ that determine the current in the SRR a line matching technique is used, where Eq. (2) is fulfilled on specific lines $C$ joining the centers of neighboring elements. ${ }^{15}$ Equations (2) and (3) lead to a system of $N$ equations for the $N$ unknown coefficients:

$$
\begin{aligned}
\int_{C}\left[\mathbf{n}(\mathbf{r}) \times \mathbf{E}^{i}(\mathbf{r})\right] \cdot d \mathbf{l}_{k}(\mathbf{r}) \\
=\sum_{j=1}^{N} \alpha_{j} \int_{C} d \mathbf{l}_{k}(\mathbf{r}) \cdot\left\{Z(\mathbf{r}) \mathbf{n}(\mathbf{r}) \times \mathbf{f}_{k}(\mathbf{r})+\mathbf{n}(\mathbf{r})\right. \\
\times\left[j \omega \iint_{S} \overline{\overline{\mathbf{G}}}_{\mathrm{AJ}}\left(\mathbf{r}, \mathbf{r}^{\prime}\right) \cdot \mathbf{f}_{j}\left(\mathbf{r}^{\prime}\right) d s^{\prime}\right. \\
\left.\left.-\frac{1}{j \omega} \nabla \iint_{S} G_{V}\left(\mathbf{r}, \mathbf{r}^{\prime}\right) \nabla \cdot \mathbf{f}_{j}\left(\mathbf{r}^{\prime}\right) d s^{\prime}\right]\right\},
\end{aligned}
$$

with $k=1,2, \ldots, N$. From the induced currents on the conductors it is an easy task to find the scattered electromagnetic field at any point in space and to compute additional observables. ${ }^{16}$ In particular, the scattering cross section (SCS) can be obtained by integrating the scattered field on a large sphere enclosing the system (typical radius $100 \lambda$ ).

\section{RESULTS}

\section{A. Individual SRR}

Figure 2 shows the scattering parameter $\mathrm{s}_{21}$ measured for a single SRR placed vertically in the middle of the waveguide, for the two different illumination polarizations depicted in Fig. 1(a). This parameter represents the amount of electromagnetic power transmitted through the guide in the presence of the SRR. To change the incident polarization, the SRR is merely rotated upon its $y$ axis [Fig. 1(a)]. For parallel polarization the magnetic field is parallel to the SRR $z$ axis and penetrates through the rings; for perpendicular polarization, the magnetic field is parallel to the SRR plane and does not penetrate through the rings. For both polarizations the electric field is parallel to the SRR $y$ axis [Fig. 1(a)].

The strongest resonance is observed for parallel polarization, as the incident field can create large currents in the SRR. In that case, the power transmitted in the waveguide is 


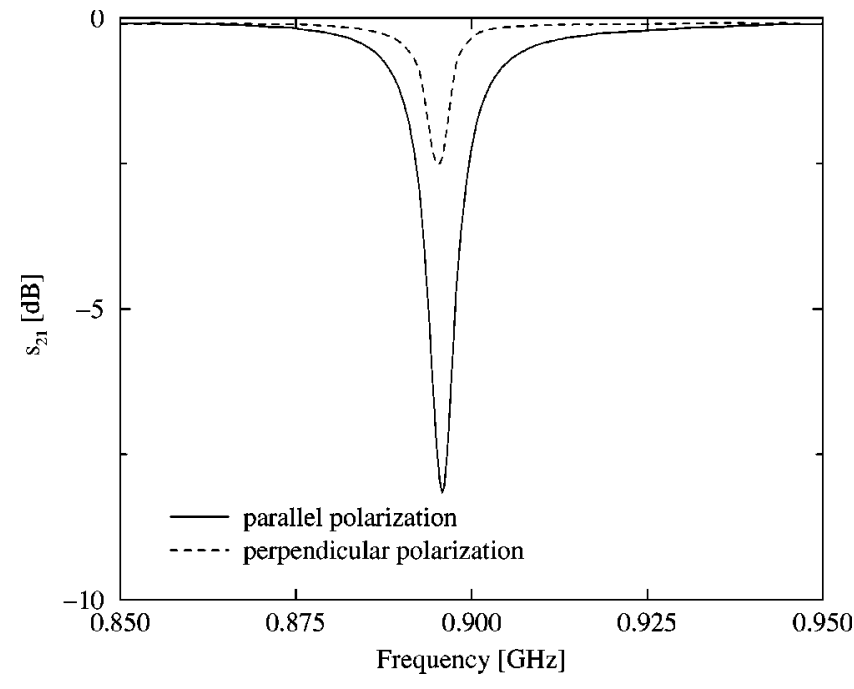

FIG. 2. Scattering parameter $s_{21}$ measured in an R9 waveguide for a single SRR illuminated in the configuration and polarizations of Fig. 1(a).

reduced by a factor of $6.5(-8.1 \mathrm{~dB})$ at $0.896 \mathrm{GHz}$. For the other polarization, a much weaker resonance is observed (Fig. 2).

If the SRR is rotated vertically around its $z$ axis as in Fig. 1(b), the resonance for parallel polarization is almost unaffected and remains very strong, while the resonance for perpendicular polarization is completely suppressed (not shown).

This behavior can be understood from the current distributions obtained in the calculations, as illustrated in Fig. 3. For parallel polarization, the incident magnetic field penetrates through the SRR and generates strong currents that flow on the rings as shown in Fig. 3(a). The currents vanish at the proximity of the gaps and are maximum at the middle of the opposite side. Therefore charges of opposite sign accumulate at the two extremities of each ring, producing a strong electric field in the gaps. The strength of this magnetic coupling only depends on the magnetic flux in the SRR and is therefore the same for both SRR orientations in Figs. 1(a) and 1(b).

External electric field can also induce currents in the SRR, as illustrated in Fig. 3(b). In that configuration the external magnetic field does not penetrate through the SRR and does not contribute to the induced currents. This electric coupling is rather weak and the induced currents about 10 times smaller in Fig. 3(b) than in Fig. 3(a), which explains the large difference in resonance strength observed in Fig. 2 for both polarizations. The electric coupling depends on the relative orientation of the SRR with respect to the electric field. When the SRR is rotated by $90^{\circ}$, minute currents with opposite directions are induced in each half-arm Fig. 3(c). In that case, charges of same sign accumulate at the two extremities of each ring; the electric field vanishes in the gap and no resonances are observed experimentally.

The spectral response of the SRR is investigated in Fig. 4 , where the current at the middle point of the external arm [point A in Fig. 1(a)] is shown as a function of the illumination frequency. The current intensity in Fig. 4(a) shows a sharp resonance for parallel polarization, and a much smaller resonance for perpendicular polarization, in good agreement with the experimental data (Fig. 2). The fact that the resonant frequency is different from the experimental one can be related to the periodic boundary conditions implicitly imposed in the experiment and shall be discussed in Sec. IV B.

The behavior of the phase of the current provides additional insights on the two resonances [Fig. 4(b)]. For perpendicular polarization, the phase changes at the resonance by approximatively $\pi$, from $+\pi / 2$ to $-\pi / 2$, as is the case in a resonant electric circuit (resistance, capacitance, and inductance in series). ${ }^{17}$ A phase shift of similar magnitude is also observed for parallel polarization. However, in that case, below the resonance frequency the phase is close to $\pi$, indicative that the current is mainly produced by the change of magnetic flux. The influence of the electric field is weak, but not negligible, otherwise the current phase would be equal to $\pi$ below the resonance. Actually, when the split ring is turned by $90^{\circ}$ around its normal axis [configuration in Fig. 1(b), with parallel polarization], so that the effect of the electric field becomes negligible, then the phase changes from $2 \pi$ below the resonance to 0 above (not shown).

The quality factor associated with the two resonances can be obtained from the SCS reported in Fig. 5. Both resonances have the same quality factor $Q=210$, although the SCS magnitude is ten times larger for the parallel polarization.

To summarize, both electric field and magnetic field induced resonances exist in SRRs, although the latter is much stronger. Note that the electric resonance is a characteristic of SRR and does not exist for uninterrupted rings. (a)

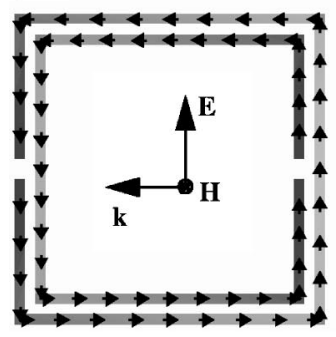

(b)

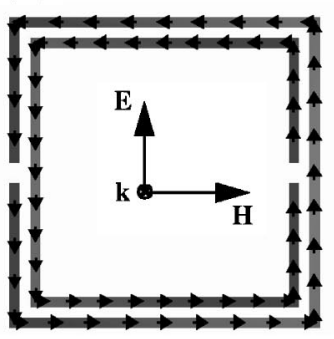

(c)

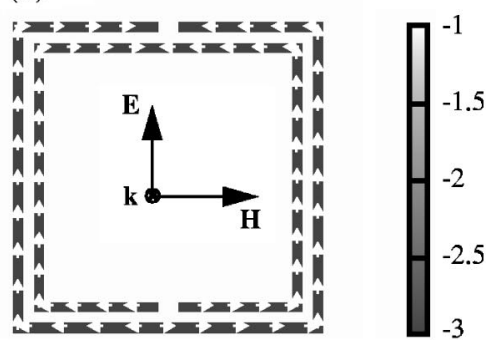

FIG. 3. Distribution of the current calculated on the rings of the resonators. The color gives the intensity (logarithmic scale) and the arrows indicate the instantaneous current direction at a specific moment in time. Two different illumination polarizations are considered: (a) parallel, (b) and (c) perpendicular polarization; and two different configurations: (a) and (b) same configuration as in Fig. 1(a), (c) same configuration as in Fig. 1(b). 

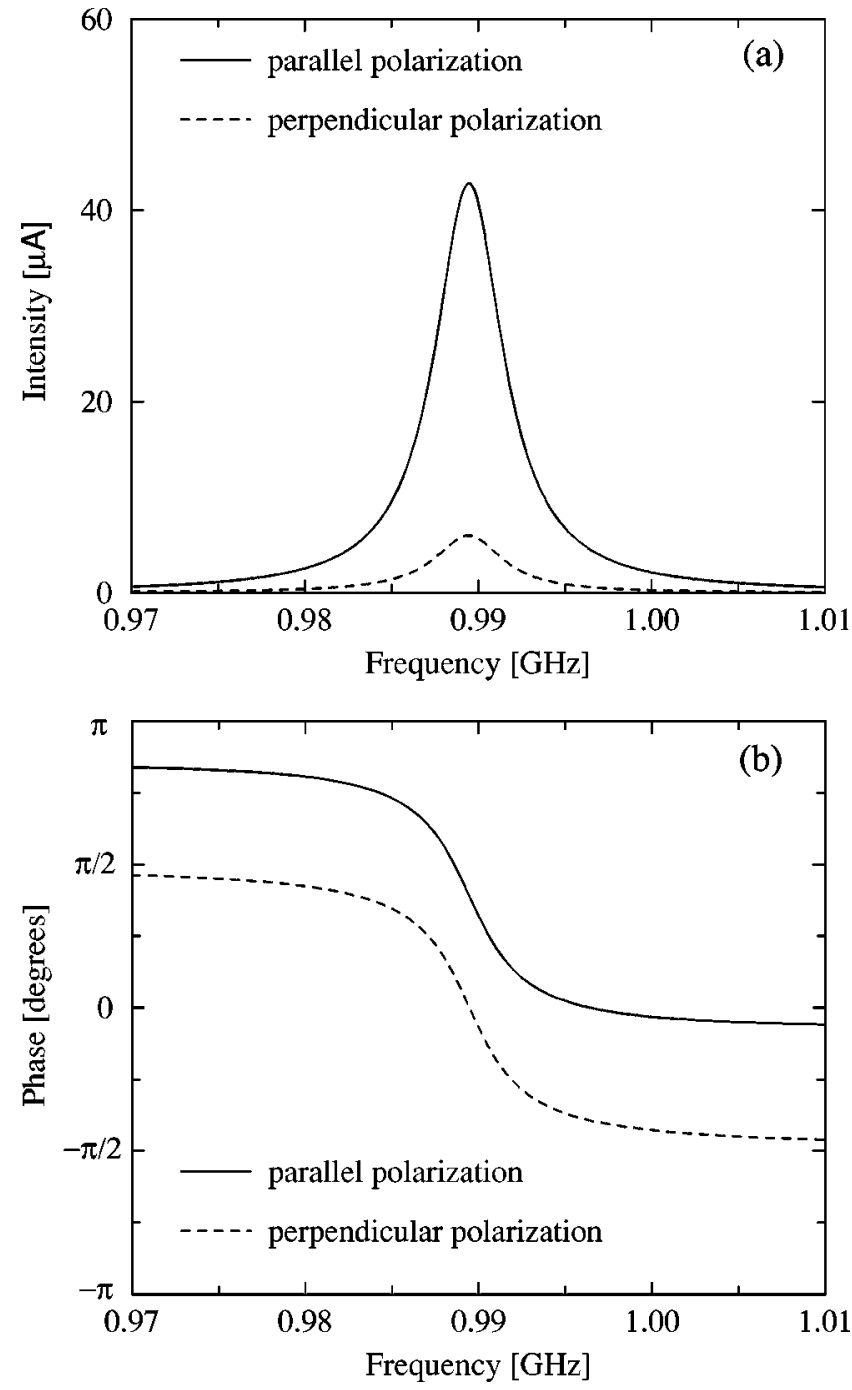

FIG. 4. Current (a) intensity and (b) phase, calculated at point A in Fig. 1(a) for two different polarizations, as a function of the illumination frequency.

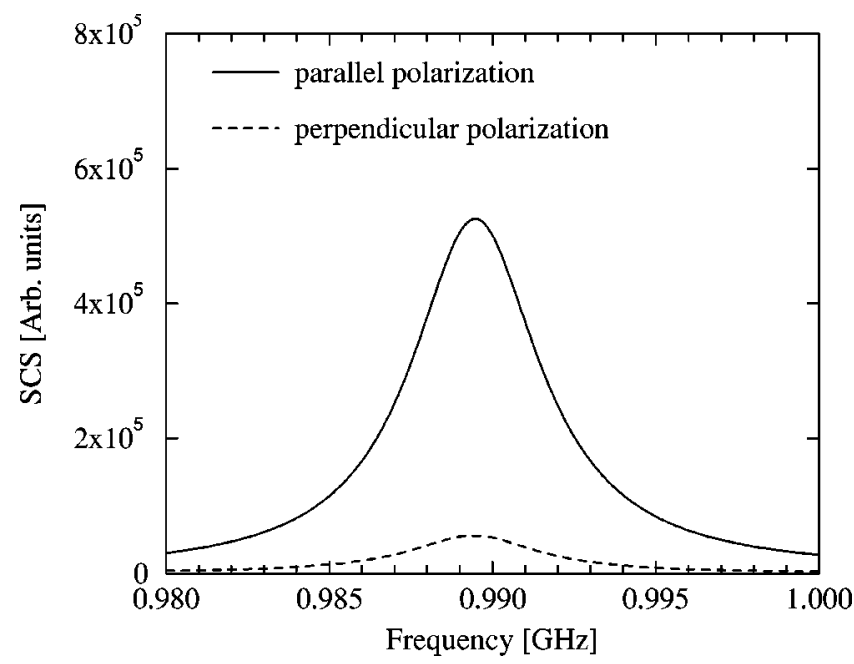

FIG. 5. Scattering cross section (SCS) computed for a single SRR illuminated with two different polarizations. (a)

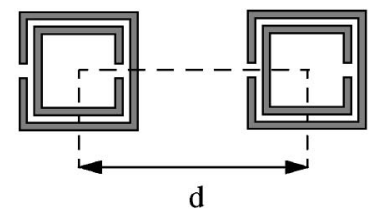

(b)
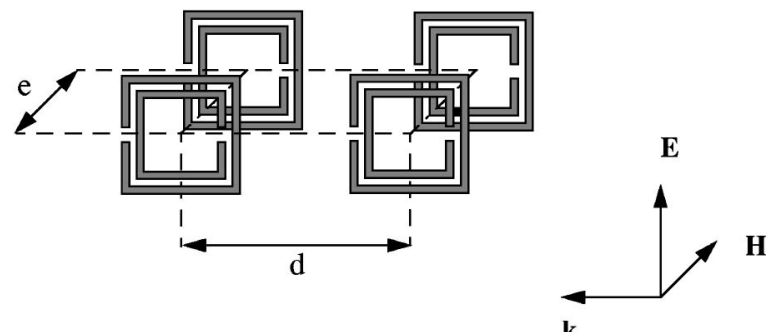

(c)

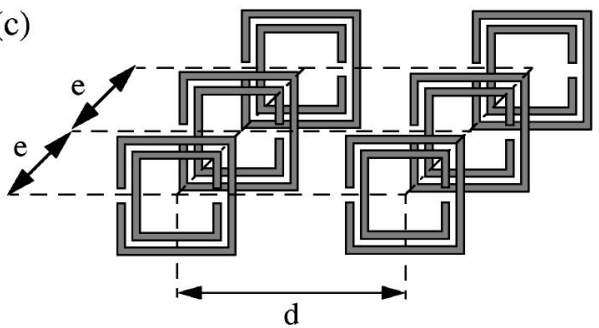

(d)
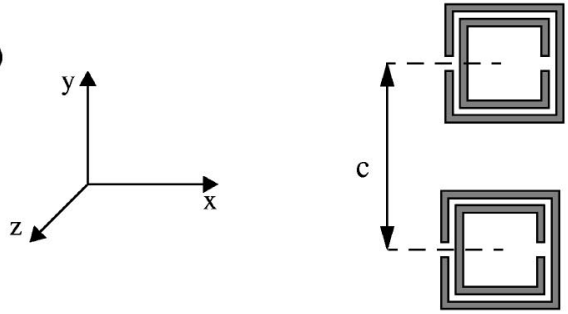

FIG. 6. Geometries considered for the study of interacting SRRs. (a) Two SRRs in a row; (b) two SRRs rows; (c) three SRRs rows, and (d) two SRRs placed side by side.

\section{B. Interacting SRRs}

In this section we study the interaction of several SRRs as a function of their geometrical arrangement. We first consider a row of parallel SRRs placed along a line in the waveguide [Fig. 6(a)]. The distance between the resonators axis is $d=\lambda_{\mathrm{m}} / 4=114 \mathrm{~mm}$, where $\lambda_{\mathrm{m}}$ is the wavelength corresponding to the $\mathrm{TE}_{10}$ mode propagating in the waveguide, at the resonance frequency of the SRRs $(0.895 \mathrm{GHz})$. For comparison with our free space numerical calculations, it is important to take into account this effective wavelength in the waveguide. $^{12}$

Figure 7 shows the $\mathrm{s}_{21}$ scattering parameters for 1, 2, 3, and 4 SRRs in the waveguide. This parameter is proportional to the transmitted power in the waveguide loaded with the SRRs. The very small discrepancy observed in the resonance frequency for the single SRR is related to our simple fabrication process which does not allow perfect control of the SRR dimensions. Each additional SRR decreases the transmitted power by approximatively $9 \mathrm{~dB}$. Like this it is possible to reduce the power transmitted through the guide by a factor of about $4000(-36 \mathrm{~dB})$, with only 4 SRRs. Furthermore, this effect remains strongly frequency selective (full 


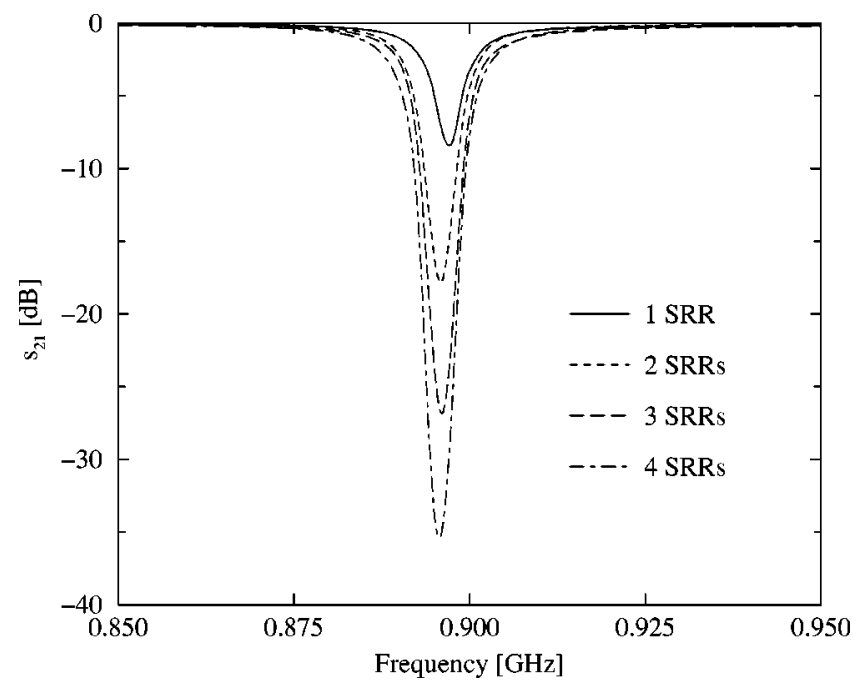

FIG. 7. Scattering parameter $s_{21}$ measured for one, two, three, and four SRRs placed in a row [Fig. 6(a)] inside an R9 waveguide.

width half maximum for 4 SRRs: $F W H M=1.02 \mathrm{MHz}, Q$ $=877$ ).

To study the coupling mechanisms between SRRs, the separation distance $d$ between two SRRs is varied [Fig. 6(a)] and the $\mathrm{s}_{21}$ parameter measured (Fig. 8). When $d$ decreases from $\lambda_{\mathrm{m}} / 2$ to $\lambda_{\mathrm{m}} / 4$, the coupling increases and the transmission through the waveguide falls to $-17.8 \mathrm{~dB}$. For a shorter separation two resonances appear $\left(d=\lambda_{\mathrm{m}} / 12\right.$, Fig. 8).

To understand this phenomenon, a series of simulations on interacting SRRs were carried out. Figure 9 shows the SCS for two SRRs with spacing $d=\lambda / 4$, for two different illumination polarizations. As for the individual SRR, the parallel polarization gives the strongest resonance; its quality factor is $Q=383$. Interesting in that figure is the slightly different resonance frequencies $f_{0}$ observed for the two polarizations: $f_{0}=0.989 \mathrm{GHz}$ for parallel polarization and $f_{0}$ $=0.991 \mathrm{GHz}$ for perpendicular polarization. This indicates

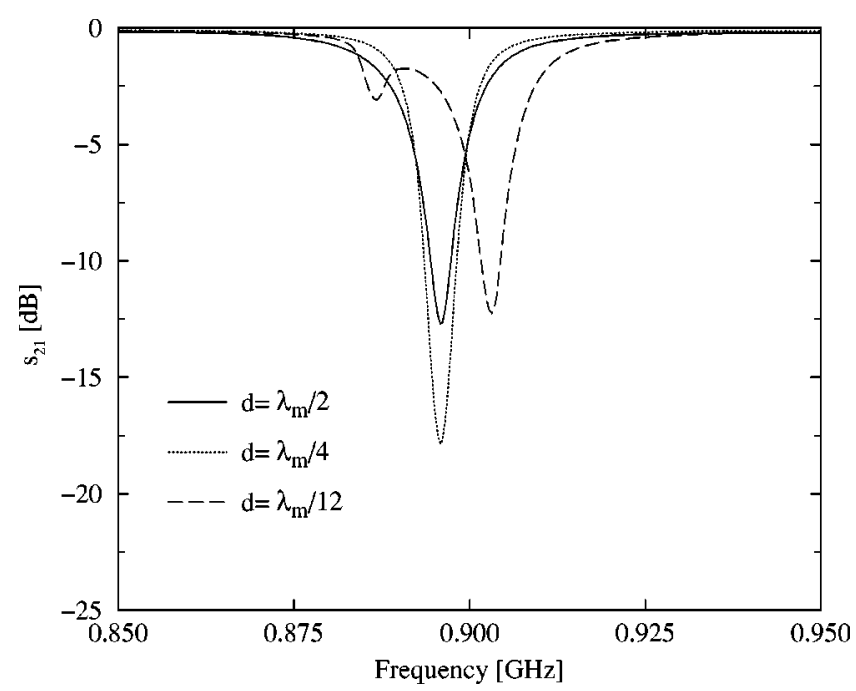

FIG. 8. Scattering parameter $s_{21}$ measured for two SRRs in a row [Fig 6(a)], for different separation distances $d$.

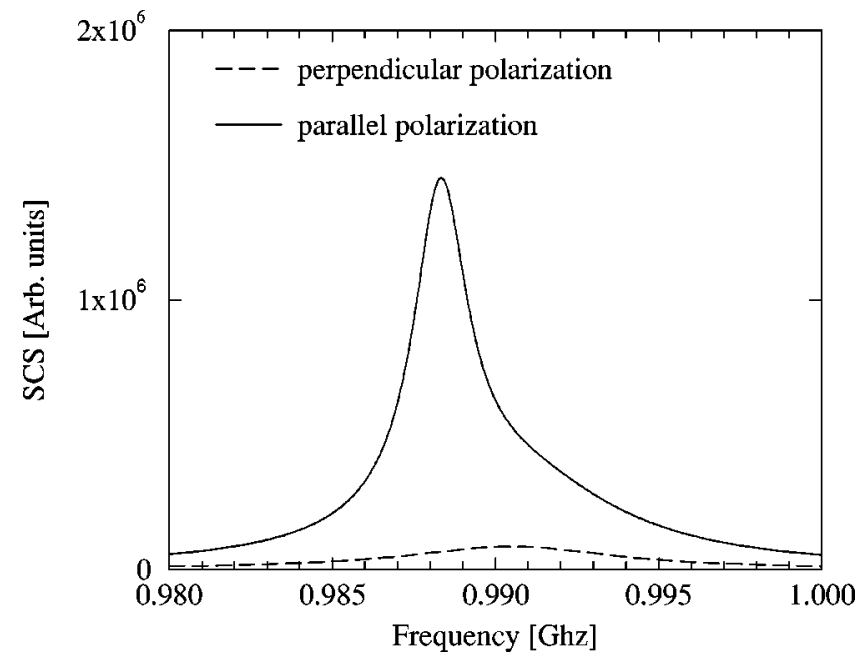

FIG. 9. Scattering cross section (SCS) computed for two SRRs with separation $d=\lambda / 4$, two different illumination polarizations.

that two different coupling modes are involved, depending on the illumination polarization.

This difference of coupling mechanisms is evidenced in Fig. 10, where the total magnetic field intensity distribution around the SRRs is reported for the two different illumination polarizations, at the corresponding resonance. Note that the magnetic field is parallel to the observation plane in Fig. 10. For parallel illumination, a loop of magnetic field that connects both SRRs is established [Fig. 10(a)]. This produces a strong magnetic coupling between the two resonators. For the other polarization, no direct magnetic field circulation is established from one SRR to the other, the field rather zig-zagging between the elements [Fig. 10(b)].

For both resonances, the field is strongly localized in space, over a volume much smaller than the corresponding radiation wavelength (Fig. 10). The magnetic field enhancement observed in Fig. 10 is in the order of 10000 for the intensity: the illumination plane wave has unit amplitude electric field, which corresponds to a magnetic field amplitude of $1 / 377 \Omega=2.6 \times 10^{-3}$. The magnetic field illumination intensity is therefore $7 \times 10^{-6}$, while the maximum intensity measured in Fig. 10 is $10^{-2}$.

To analyze the additional resonance observed experimentally in Fig. 8 for short separation distances $d$, calculations of the SCS for two SRRs with varying spacing $d$ were performed. These results are summarized in Fig. 11 where we report the quality factor $Q$ and the main resonance frequency $f_{0}$, parameterized by the separation distance $d$ for $d=\lambda / 8 \ldots \lambda$. We can identify two regions in that plot: for $d$ $=\lambda / 4 \ldots \lambda$ (inset in Fig. 11), the resonance frequency remains around $f_{0}=0.989 \mathrm{GHz}$ and the quality factor around $Q$ $=200$. For shorter separation distances, $Q$ suddenly explodes and the resonance frequency shifts towards lower values (Fig. 11). It is in this region that a second resonance appears (Fig. 8).

The transition between these two regions corresponds to the splitting of the main resonance into two resonances, as illustrated in Fig. 12. These calculations show that for separation distances $d<\lambda / 4$, the main resonance is strongly 
(a)

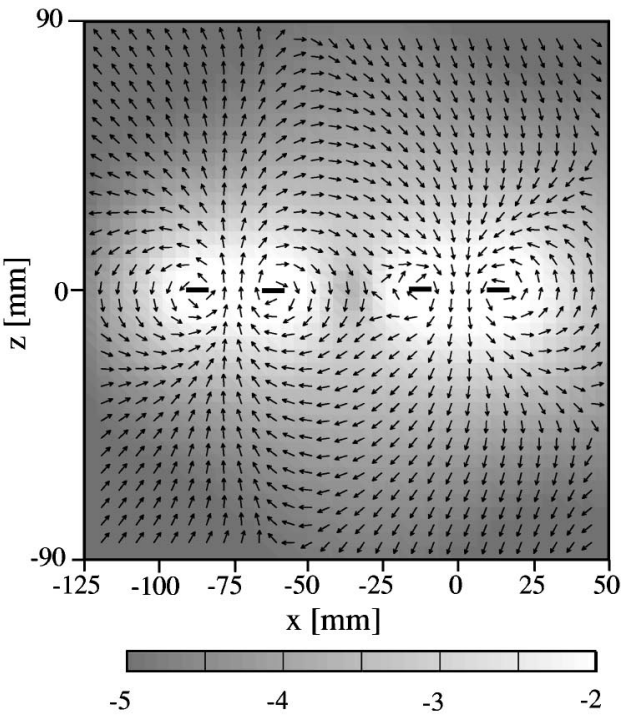

(b)

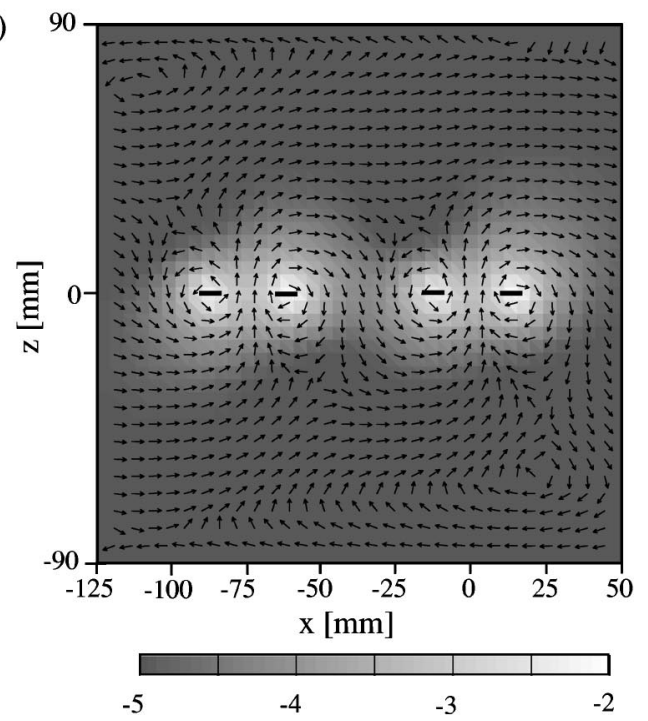

FIG. 10. Total magnetic field distribution calculated around two SRRs with spacing $d=\lambda / 4$, at the resonance frequency $f_{0}$. Two different illumination polarizations are considered: (a) Parallel polarization $\left(f_{0}=0.989 \mathrm{GHz}\right)$ and (b) perpendicular polarization $\left(f_{0}=0.991 \mathrm{GHz}\right)$. The distributions are computed in an $x y$ plane through the middle of the structures $(y=0)$. The grayscale represents the magnetic field intensity on a logarithmic scale and the arrows give the instantaneous projection of the magnetic field orientation on the observation plane. The black segments show the locations of the SRRs.

shifted towards lower frequencies and an additional, broader resonance, appears at a higher frequency. This effect is particularly visible for a very short separation $(d=\lambda / 8$, Fig. 12). The main resonance has the same magnetic field pattern as in Fig. 10(a) and the second, higher frequency, resonance has that of Fig. 10(b). Note also that the main resonance becomes extremely narrow, leading to a very large quality factor $(Q=767$ for $d=\lambda / 6$ and $Q=1143$ for $d=\lambda / 8)$.

This resonance splitting is in good qualitative agreement with our experimental data (compare Figs. 8 and 12), although the very sharp lower frequency resonance is not well resolved in the experiment (Fig. 8). Simulations show that this resonance is extremely sensitive to defects in the system

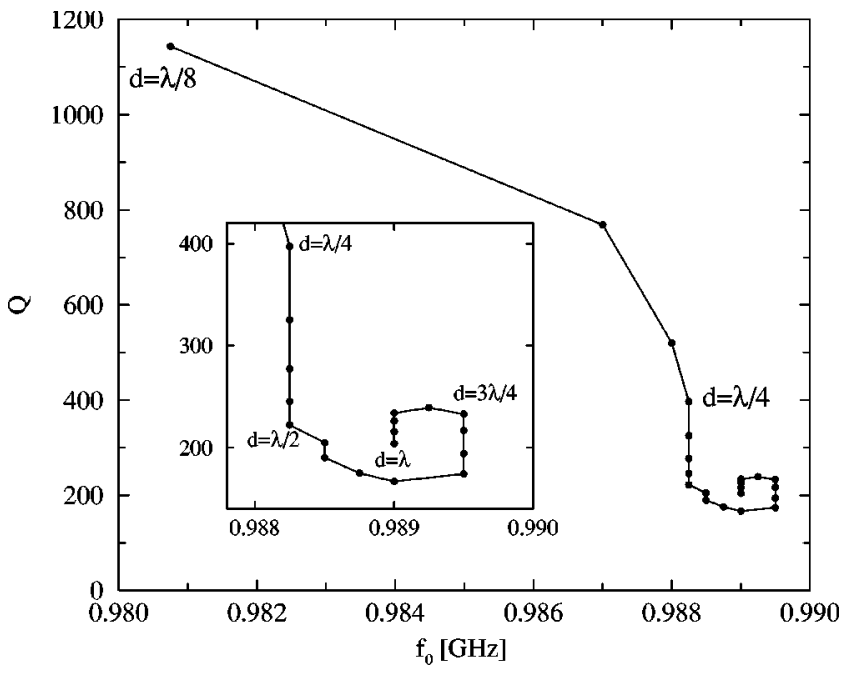

FIG. 11. Quality factor $Q$ and frequency $f_{0}$ of the main resonance calculated for two interacting SRRs, parameterized by the spacing $d$ between the SRRs.

and it is possible that the glue used to stick the aluminum foil on the substrate disturbs this resonance.

This enhanced resonance phenomenon as a function of the separation distance is also in good agreement with data on periodic SRRs array, ${ }^{11}$ where the coupling between SRRs augments when the fractional cell volume occupied by the resonant structures increases, i.e., when the spacing between neighboring SRRs decreases.

When additional rows of SRRs are added along the $z$ direction [Figs. 6(b) and 6(c)], the resonance frequencies $f_{0}$ are shifted to lower values, as illustrated in Fig. 13 for two different separation distances $d$. The computed $f_{0}$ values become then much closer to the experimental values. This is in good agreement with the fact that in the experiment the waveguide implicitly imposes periodic boundary conditions, so that the physical system effectively measured represents many rows of SRRs. Notice in Fig. 13 that the additional frequency shift decreases when more SRR rows are added;

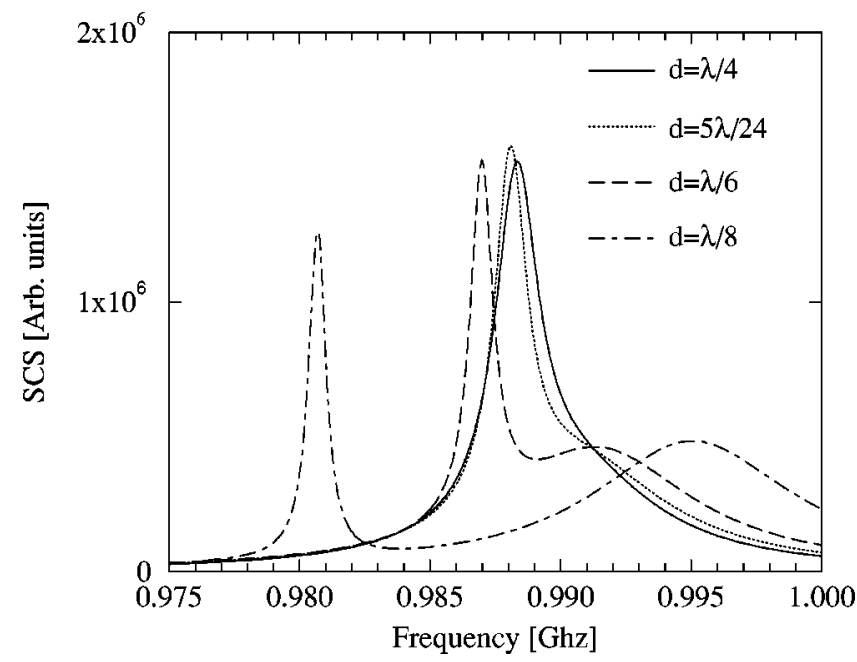

FIG. 12. Scattering cross section (SCS) computed for two SRRs with small separation distances $d$ [Fig. 6(a)]. 


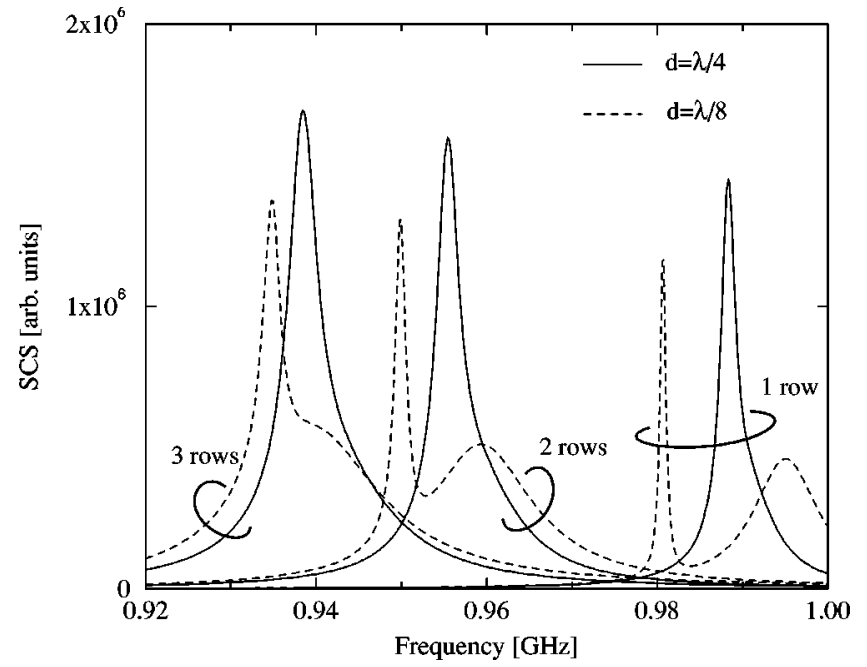

FIG. 13. Scattering cross section (SCS) computed for 1, 2, and 3 rows of SRRs resonators [Figs. 6(a)-6(c)]. Two different separation distances $d$ have been selected; the inter-row spacing is $c=20 \mathrm{~mm}$.

further simulations indicate that the resonance frequency already converges for about eight rows. The resonance width also broadens when additional rows are introduced (Fig. 13). Correspondingly the quality factor decreases: $Q=397$ (one row), $Q=245$ (two rows), and $Q=184$ (three rows).

The inter-row spacing $e$ also influences the resonances, as illustrated in Fig. 14 for two rows with two SRRs each. For small spacing, $e<30 \mathrm{~mm}$, the resonance shifts towards lower frequencies and becomes narrower. The quality factor increases accordingly: $Q=212(e=60 \mathrm{~mm}), Q=220(e$ $=30 \mathrm{~mm}), Q=243(e=20 \mathrm{~mm}), Q=269(e=15 \mathrm{~mm}), Q$ $=323(e=10 \mathrm{~mm})$. Short inter-row separation enhances the coupling between SRRs, which is in good agreement with previous results on infinite periodic arrays of SRRs or cylindrical structures. ${ }^{11}$

As last coupling mechanism we investigate two SRRs placed side by side, as illustrated in Fig. 6(d). The corre-

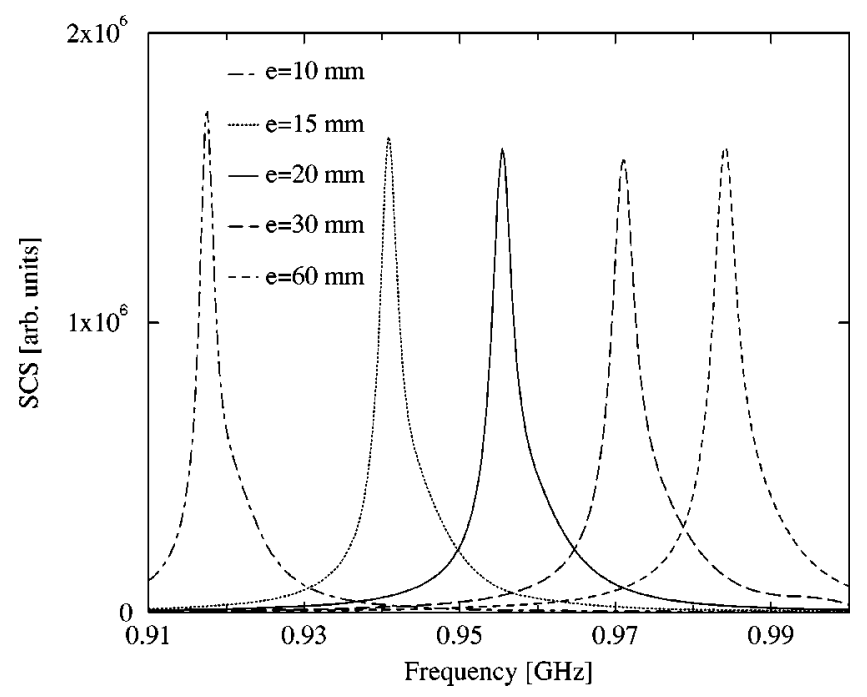

FIG. 14. Scattering cross section (SCS) calculated for the configuration of Fig. 6(b), for different distances $e$ between the rows. The separation in a row is $d=\lambda / 4$.

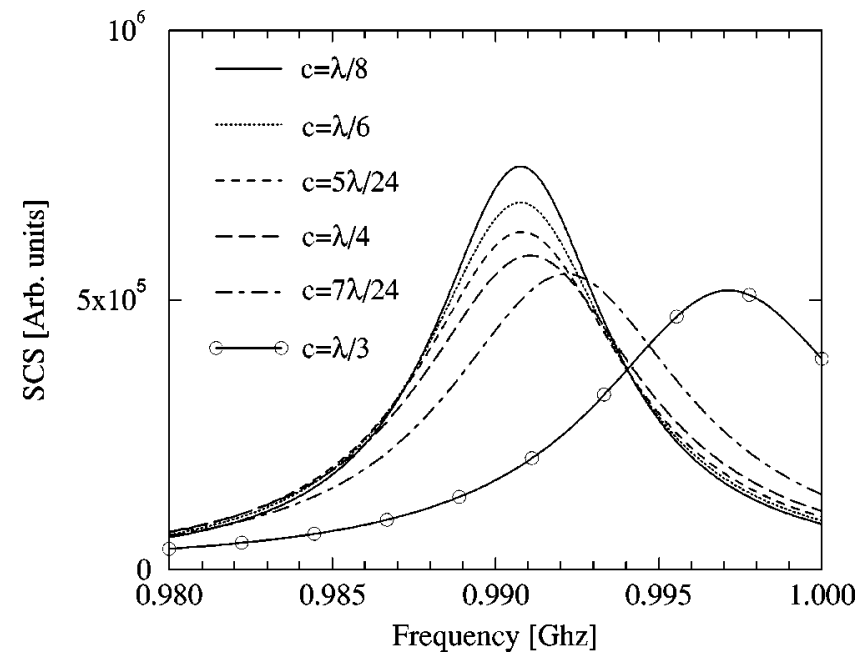

FIG. 15. Scattering cross section (SCS) computed for the configuration of Fig. 6(d), for different lateral separation distances $c$.

sponding resonances are shown in Fig. 15 as a function of the lateral separation distance $c$. This resonance is not very strong. In particular its quality factor only varies from $Q$ $=110(c=\lambda / 8)$ to $Q=153(c=\lambda / 3)$ and is always smaller than the one associated with a single $\operatorname{SRR}(Q=210)$. When the lateral separation distance $c$ decreases the resonance frequency shifts towards lower values, as was the case for the longitudinal separation $d$. However, no resonance splitting occurs when the SRR are placed side by side, even for extremely small separation $c$ (Fig. 15).

\section{CONCLUSIONS}

The electromagnetic resonances in SRRs have been investigated experimentally and numerically, at frequencies around $1 \mathrm{GHz}$. A good agreement was obtained between the two.

Our results indicate that the electromagnetic phenomena that take place in such structures are quite subtle. For an individual SRR, the magnetic effects are dominant, with strong currents induced in the rings when the magnetic field penetrates through the rings. Contrary to uninterrupted rings, the electric field can also induce currents in a SRR. This effect is however about 10 times weaker than the magnetic one and strongly depends on the exact orientation of the rings arms with respect to the illumination electric field.

At the resonance, the magnetic field is strongly localized in a very small volume at the vicinity of the SRR. Its intensity is strongly enhanced, in the order of $10000 \times$ the illumination magnetic field intensity.

In a rectangular waveguide, a SRR acts as efficient filter element. This effect can be reinforced when several SRRs are placed in a row along the propagation direction of the electromagnetic field. In that configuration, strong coupling between neighboring SRRs occurs for small separation distances. This coupling is associated with a magnetic field circulation that is established between neighboring elements. This type of coupling, with SRRs aligned with the field propagation direction, appears to be the strongest one and 
when SRRs are placed side by side or in different layers, the quality factor of the overall resonance decreases.

The resonance frequency $f_{0}$ depends noticeably on the separation distance between SRRs and shifts towards lower values when the separation distance in any direction decreases. Our results show variations of $f_{0}$ in the order of several ten $\mathrm{MHz}$, for distance variations smaller than $\lambda / 2$. This sensitivity to the geometrical arrangement imposes constraints on the accuracy with which SRR arrays must be fabricated. On the other hand, it also offers the possibility to tune the structure and place the resonance frequency at a specific value. This is important when SRRs are combined with a plasmonic medium to produce a left-handed metamaterial. ${ }^{1}$ Since a negative permittivity can be obtained on a large frequency range, the magnetic resonance, which determines the narrow range where a negative permeability is observed, defines the left-handed band in the material.

The resonance characteristics of a SRR array depend on the number of elements. Our data indicate that they already converge with about eight elements in each direction, which is important for the implementation of SRRs in a practical device with finite size.

\section{ACKNOWLEDGMENTS}

The authors are most grateful to $\mathrm{H}$. Benedickter for his efficient support during the fabrication and the measure- ments. It is a pleasure to acknowledge stimulating discussions with J.B. Pendry, S. Schultz, and D.R. Smith. This work was supported by the Swiss National Science Foundation, the Research Board of ETHZ, and AFOSR (Grant No. F496200110440).

${ }^{1}$ D. R. Smith et al., Phys. Rev. Lett. 84, 4184 (2000).

${ }^{2}$ R. A. Shelby, D. R. Smith, S. C. Nemat-Nasser, and S. Schultz, Appl. Phys. Lett. 78, 489 (2001).

${ }^{3}$ R. A. Shelby, D. R. Smith, and S. Schultz, Science 292, 77 (2001).

${ }^{4}$ V. G. Veselago, Sov. Phys. Usp. 10, 509 (1968).

${ }^{5}$ J. B. Pendry, Phys. Rev. Lett. 85, 3966 (2000).

${ }^{6}$ R. Ruppin, Solid State Commun. 116, 411 (2000).

${ }^{7}$ R. Ruppin, J. Phys. C 13, 1811 (2001).

${ }^{8}$ C. Caloz, C.-C. Chang, and T. Itoh, J. Appl. Phys. 90, 5483 (2001).

${ }^{9}$ J. B. Pendry, A. J. Holden, W. J. Stewart, and I. Youngs, Phys. Rev. Lett. 76, 4773 (1996).

${ }^{10}$ J. B. Pendry, A. J. Holden, D. J. Robbins, and W. J. Stewart, J. Phys. C 10, 4785 (1998)

${ }^{11}$ J. B. Pendry, A. J. Holden, D. J. Robbins, and W. J. Stewart, IEEE Trans. Microwave Theory Tech. 47, 2075 (1999).

${ }^{12} \mathrm{~F}$. Gardiol, Introduction to Microwaves (Artech House, Dedham, MA, 1984).

${ }^{13}$ L. Barlatey, J. R. Mosig, and T. Sphicopoulos, IEEE Trans. Antennas Propag. 38, 608 (1990).

${ }^{14}$ M. Paulus, P. Gay-Balmaz, and O. J. F. Martin, Phys. Rev. E 62, 5797 (2000).

${ }^{15}$ P. Gay-Balmaz and J. R. Mosig, Int. J. Microwave Millimeter Wave CAE 3, 330 (1997).

${ }^{16}$ O. J. F. Martin and N. B. Piller, Phys. Rev. E 58, 3909 (1998).

${ }^{17}$ P. Feynman, R. B. Leighton, and M. Sands, Lectures on Physics (AddisonWesley, Reading, MA, 1963), Vol. 1. 